\title{
s \\ Detailed balance theory of excitonic and bulk heterojunction solar cells
}

\author{
Thomas Kirchartz, ${ }^{1, *}$ Julian Mattheis, ${ }^{2}$ and Uwe Rau ${ }^{1}$ \\ ${ }^{1}$ IEF5-Photovoltaik, Forschungszentrum Jülich, 52425 Jülich, Germany \\ ${ }^{2} Q$-Cells AG, Sonnenallee 17-21, 06766 Bitterfeld-Wolfen, Germany \\ (Received 25 July 2008; revised manuscript received 24 November 2008; published 29 December 2008)
}

\begin{abstract}
A generalized solar cell model for excitonic and classical bipolar solar cells describes the combined transport and interaction of electrons, holes, and excitons in accordance with the principle of detailed balance. Conventional inorganic solar cells, single-phase organic solar cells and bulk heterojunction solar cells, i.e., nanoscale mixtures of two organic materials, are special cases of this model. For high mobilities, the compatibility with the principle of detailed balance ensures that our model reproduces the Shockley-Queisser limit irrespective of how the energy transport is achieved. For less ideal devices distinct differences become visible between devices that are described by linear differential equations and those with nonlinear effects, such as a voltagedependent collection in bipolar $p$ - $i$ - $n$-type devices. These differences in current-voltage characteristics are also decisive for the validity of the reciprocity theorem between photovoltaic quantum efficiency and electroluminescent emission. Finally, we discuss the effect of band offset at the heterointerface in a bulk heterojunction cell and the effect of the average distances between these heterointerfaces on the performance of a solar cell in order to show how our detailed balance model includes also these empirically important quantities.
\end{abstract}

DOI: 10.1103/PhysRevB.78.235320

PACS number(s): 84.60.Jt, 72.80.Le, 78.60.Fi

\section{INTRODUCTION}

More than 50 years after the introduction of the first device, ${ }^{1}$ solar cells and solar modules contribute an increasing and more and more sizeable share to the world's demand for clean and renewable electrical energy. More than $90 \%$ of the actual solar module production uses crystalline siliconbased $p n$-junction solar cells, i.e., the same type as the pioneer device from 1954. Within these years, our understanding of the device, its working principles, and its processing has obviously achieved a high degree of maturity. ${ }^{2,3}$ During the last 15 years, a variety of new types of solar cells ${ }^{4,5}$ based on quite different materials and concepts such as the dye-sensitized $^{6}$ or the bulk heterojunction (BHJ) (Ref. 7) solar cell have been introduced. As these devices use, e.g., electrolytes, semiconductor nanoparticles, ${ }^{8-15}$ organic dyes, ${ }^{16-20}$ and/or organic semiconductors as functional elements, the basic physics underlying light absorption, charge separation, and energy generation differs considerably from what is well established for classical $p n$-junction solar cells made from inorganic semiconductors. Recently, it has been proposed $^{21,22}$ to distinguish between two different concepts of solar cells, namely, the classical inorganic solar cells and the excitonic solar cells usually made from organic absorber materials. However, the overall functionality in all solar cells, namely, the generation of electrical power from solar light, is identical, and there should be a common theory that is valid for all devices on a certain level of abstraction from physical details.

Obviously, the detailed balance theory of the maximum photovoltaic energy conversion efficiency by Shockley and Queisser (SQ) (Ref. 23) is an approach that accounts for all types of (single junction) solar cells as a limiting situation. The generality of the SQ approach results from the fact that only the detailed balance pair light absorption and light emission are considered. This implies that all details of excitation, charge separation, and transport inside the device are ne- glected by the postulate that all absorption of light leads to the generation of charge carriers that under short circuit conditions are all collected by the electrical terminals of the device. The principle of detailed balance then ensures that such a perfect solar cell is also a perfect light emitting diode (LED) and the balance between light absorption and emission defines the radiative efficiency limit. ${ }^{23}$ Thus, the SQ theory essentially looks at the solar cell from the outside. This neglect of internal details makes up the elegance of the SQ approach but, at a first glance, disconnects this theory from any model designed to describe the internal operation of the solar cell.

The present paper uses the basic concept behind the SQ theory - the principle of detailed balance - to develop a generalized model of solar cells, considering both excitons and free charge carriers as well as $p n$-junction-type and $p-i$ - $n$-junction-type device geometries. The basic ingredients are (i) light absorption and creation of an exciton as well as radiative recombination and photon emission, (ii) exciton dissociation at an interface or in the bulk and the corresponding inverse process, the recombination of an electron/hole pair in an exciton, and (iii) drift-diffusion transport of electrons and holes as well as diffusive transport of excitons.

The compliance of our model with the principle of detailed balance ensures that for high mobilities and solely radiative recombination, we obtain the same photovoltaic performance of our device as the one following from the SQ limit no matter whether transport is predominantly excitonic or bipolar and no matter whether our device is $p n$-type or $p$ - $i$ - $n$-type. Proceeding to devices with nonideal transport, we identify differences between the cases of predominantly excitonic and predominantly bipolar transport in $p$ - $i$ - $n$-type devices. Since the built-in field in $p$ - $i$ - $n$-type devices depends on the applied voltage, collection and injection of charged particles such as electrons and holes will also be a function of voltage if mobilities are low. This voltage dependence is not only visible in simulated current-voltage curves but also 
in electroluminescence (EL) spectra. We show that the validity of the reciprocity ${ }^{24}$ between EL and solar cell quantum efficiency directly correlates with the voltage dependence of carrier collection and injection. For the cases of either high mobilities or excitonic transport, no voltage dependence is visible and the reciprocity relation is valid. In addition to the cases of excitonic and bipolar solar cells, we also discuss how to incorporate the concept of bulk heterojunction solar cells into our model. Finally, we explain how the effects of band offsets at the heterointerface and the morphology of the blend in the bulk heterojunction cell are implicitly contained in the principle of detailed balance.

The paper is organized as follows. We start with a discussion of our model and show how the choice of the boundary conditions allows us to simulate both $p n$-type and $p$ - $i$ - $n$-type solar cells. The theoretical part is finished by explaining how to implement bulk heterojunction solar cells in the scheme of our model and how the reciprocity between EL and solar cell quantum efficiency is defined. The discussion of the results starts with current-voltage simulations of different solar cells and shows that for the same cells how quantum efficiency and electroluminescence are affected by nonideal transport. Eventually, the dependence of photovoltaic parameters on band offset and blend morphology of a BHJ solar cell are shown.

\section{MODEL}

\section{A. Excitonic and bipolar solar cells}

To be compatible with the detailed balance theory of Shockley and Queisser, the absorption of photons emitted by the device itself has to be taken into account. ${ }^{25}$ This effect of optical coupling in the absorber is usually referred to as photon recycling and leads to a homogenization of the carrier distribution. Incorporation of the above-mentioned processes in a numerical model results in four coupled differential equations to be solved, namely, the Poisson equation $\Delta \varphi$ $=-\rho / \varepsilon$, relating the electrical potential $\varphi$ to the space charge $\rho$ and the dielectric constant $\varepsilon$, and three continuity equations, one for the exciton concentration $\chi$ given by

$$
D_{\chi} \frac{d^{2} \chi}{d x^{2}}=\frac{\chi}{\tau_{D}}+\frac{\chi}{\tau_{r}}-R_{\chi} n p-g_{\chi},
$$

one for the free-electron concentration $n$,

$$
D_{n} \frac{d^{2} n}{d x^{2}}+F \mu_{n} \frac{d n}{d x}=-\frac{\chi}{\tau_{D}}+R_{\chi} n p,
$$

and one for the free-hole concentration $p$,

$$
D_{p} \frac{d^{2} p}{d x^{2}}-F \mu_{p} \frac{d p}{d x}=-\frac{\chi}{\tau_{D}}+R_{\chi} n p .
$$

Here, $g_{\chi}$ is the optical generation rate for excitons, $R_{\chi}$ is the recombination rate of free carriers leading to the creation of excitons, $\tau_{D}$ is the bulk dissociation lifetime of the excitons, $\tau_{r}$ is the radiative lifetime of the excitons, $F$ is the electric field, $\mu_{\chi / n / p}$ are the mobilities, and $D_{\chi / n / p}=\mu_{\chi / n / p} k T / q$ are the diffusion constants according to Einstein's equation, where
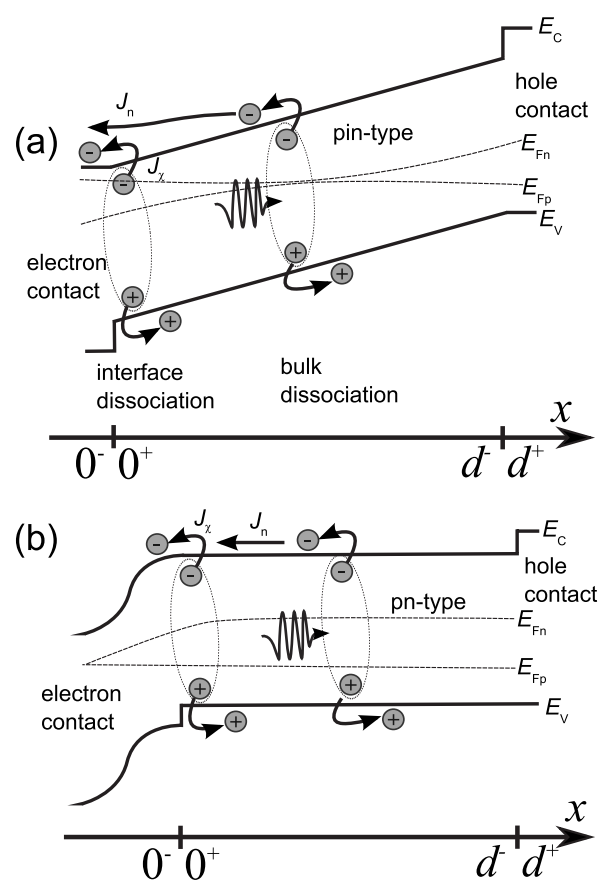

FIG. 1. Scheme of the (a) $p-i-n$-junction device and the (b) $p n$-junction device. For both types the photogenerated exciton can be either split in the bulk (bipolar case) or at the junction (excitonic case) of the device. Quasi-Fermi levels $E_{F n}$ and $E_{F p}$ for electrons and holes levels are schematically drawn for the case of illuminated short circuit conditions and finite mobilities.

$k T$ is the thermal energy and $q$ is the elementary charge. Here, we make the simplification that photons only create excitons directly, while free charge carriers are created always via the intermediate step of exciton generation followed by exciton dissociation. Although a direct creation of free carriers is not possible, the situation is covered by infinitely short dissociation lifetimes $\tau_{D}$. Note that an electrical field is present only in case of a $p-i-n$-junction as sketched in Fig. 1(a), whereas we have no electric field in the bulk of the absorber of a $p n$-junction device [Fig. 1(b)]. In case of the $p n$-type device, we only solve the differential Eqs. (1)-(3) in the neutral $p$ layer, while the $p n$-junction itself and the $n$ layer define the boundary condition, which will be explained in Sec. II B. Similarly for the $p-i-n$-junction device, we only solve the differential equations in the intrinsic region, while the $p$-type and the $n$-type layers at both sides determine the boundary conditions.

Equations (1)-(3) already contain the detailed balance relations between the different processes, meaning that the rates are connected to the equilibrium concentrations $\chi_{0}, n_{0}$, and $p_{0}$ of excitons, electrons, and holes by the fact that the probability of every process must equal that of its inverse process in thermodynamic equilibrium. Thus, radiative recombination is linked to absorption via the van RoosbroeckShockley equation, ${ }^{26}$

$$
\chi_{0} / \tau_{r}=\int \alpha_{\chi}(E) \phi_{\mathrm{bb}}(E) d E,
$$

where $\alpha_{\chi}(\mathrm{E})$ denotes the absorption coefficient resulting from the generation of excitons, $E$ denotes the photon en- 
ergy, and $\phi_{\mathrm{bb}}(E)$ denotes the black body radiation. Detailed balance between dissociation and recombination of excitons and electron-hole pairs leads to

$$
R_{\chi} n_{0} p_{0}=\chi_{0} / \tau_{D}
$$

Similarly, at the front or the back surface we allow for dissociation of an exciton into an electron in the respective junction and a hole that remains in the bulk of the absorber, as well as the complementary process, dissociation into a hole in the junction and an electron in the bulk. The rates for this type of charge separation that is thought to be the dominant one in excitonic solar cells are determined by the detailed balance relations,

$$
H_{n} n_{j 0} p_{b 0}=S_{\chi n} \chi_{0}
$$

and

$$
H_{p} n_{b 0} p_{j 0}=S_{\chi p} \chi_{0},
$$

where $H_{n / p}$ are the cross recombination rates of electrons or holes in the junction with holes or electrons in the bulk of the absorber but directly next to the contact. The equilibrium concentrations of electrons and holes in the bulk junction are denoted by $n_{b / j 0}$ and $p_{b / j 0}$ and the dissociation velocities for the excitons by $S_{\chi n}$ and $S_{\chi p}$. In the nonequilibrium situation, this interface dissociation and recombination process defines the boundary condition for the diffusion equation [Eq. (1)]. Assuming that at the left contact (Fig. 1) the dissociation of an exciton leads to an electron in the junction and a hole in the bulk, the exciton current density $j_{\chi}$ reads

$$
j_{\chi}=H_{n} n_{j} p_{b}-S_{\chi n} \chi .
$$

Accordingly, at the right contact only dissociation of an exciton into a hole in the junction and an electron in the bulk is allowed. Thus, we have

$$
j_{\chi}=-H_{p} n_{b} p_{j}+S_{\chi p} \chi .
$$

Since at the left contact the dissociation of an exciton leads to the generation of a hole in the bulk, we have here the boundary condition for holes,

$$
j_{p}=-H_{n} n_{j} p_{b}+S_{\chi n} \chi .
$$

For electrons, we consider at this contact the possibility of extraction and injection to obtain

$$
j_{n}=S_{n}^{*} n_{j}-S_{n} n_{b},
$$

where the rate constants $S_{n}$ and $S_{n}^{*}$ have the dimension of a (collection or injection) velocity and are interlinked by the requirements of detailed balance via

$$
S_{n}^{*} n_{j 0}=S_{n} n_{b 0} \text {. }
$$

At the right contact we have for symmetry reasons

$$
j_{n}=H_{p} n_{b} p_{j}-S_{\chi p} \chi
$$

and

$$
j_{p}=-S_{p}^{*} p_{j}+S_{p} p_{b}
$$

with

$$
S_{p}^{*} p_{j 0}=S_{n} p_{b 0} .
$$

Note that for simplicity we assume throughout this paper that only the electron contact at the left is active in dissociating excitons. Accordingly, we set $H_{p}=0$ and $S_{\chi p}=0$ such that we get $j_{\chi}=j_{n}=0$ at the right contact because of Eqs. (9) and (13).

Note also that throughout this paper we use the symbol $j$ for particle (excitons, electrons, and holes) current densities per unit junction area, whereas we use $J$ for electrical current densities per unit solar cell area. As long as we are dealing with a "normal" heterojunction and not a bulk heterojunction, we have $J=q j_{p}(x=d)=-q\left[j_{n}(x=0)+j_{\chi}(x=0)\right]$, with the coordinate $x$ as shown in Fig. 1 .

\section{B. $p n$-type and $p$-i-n-type solar cells}

Figures 1(a) and 1(b) schematically show (a) a $p$ - $i$ - $n$-junction device and (b) a $p n$-junction device with a sketch of both the excitonic as well as the bipolar photocurrent collection pathways. For the $p-i-n$-junction device the absorber material is embedded between the $p$-type and the $n$-type contacts. Thus, the fundamental capacitor that serves to electrostatically accommodate the open circuit voltage ${ }^{27}$ is defined by the entire device. The built-in potential difference between the electron and hole contacts in the $p$ - $i$ - $n$-type device is given by

$$
V_{\mathrm{bi}}=V_{\mathrm{bi}, 0}-V,
$$

where $V$ is the applied voltage and $V_{\mathrm{bi}, 0}$ is the equilibrium built-in voltage. Equation (16) implies that application of a voltage $V$ reduces the potential between the left and right edges of the absorber and the electrical field $F$ in the absorber. The reduced field then leads to an injection of electrons via the left contact and of holes via the right contact without a change in $n_{j}$ or $p_{j}$ from their equilibrium values $n_{j 0}$ and $p_{j 0}$ in Eq. (11) or (14). A part of the holes injected from the right contact arrive at the left contact where their increased concentration $p_{b}$ triggers the generation of excitons [Eq. (8)].

In contrast, for the $p n$-junction the external voltage builds up across the space charge region which is here put outside the absorber [cf. Fig. 1(b)]. The concentration $n_{j}$ of electrons provided by the junction then follows the applied voltage $V$ exponentially, i.e.,

$$
n_{j}=n_{j 0} \exp (q V / k T) .
$$

This increased number $n_{j}$ of electrons provided by the junction triggers now both the generation of excitons at the interface, via Eq. (8), as well as the injection of electrons, via Eq. (11).

\section{Bulk heterojunction solar cells}

Efficient organic solar cells do not consist of a single absorber material like most inorganic solar cells. Instead, the whole bulk is made up of an intimate mixture of two materials, creating a heterojunction everywhere in the absorber. ${ }^{28-35}$ Figure 2 shows a schematic and ordered version of this BHJ-type device. ${ }^{36}$ There are different possibilities to model BHJ solar cells, either via complex and detailed 


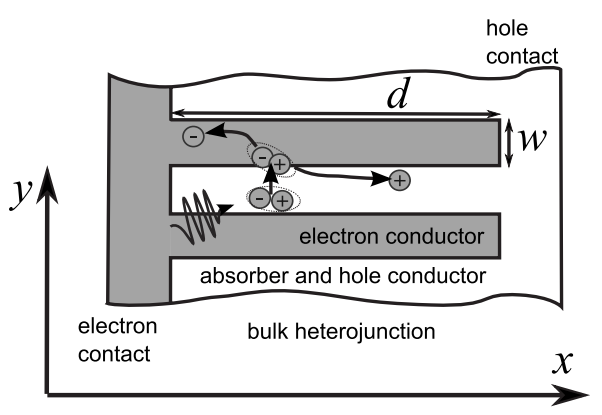

FIG. 2. Scheme of a BHJ solar cell. Bulk dissociation of excitons is interpreted in terms of BHJ devices as the combined process of diffusion to and subsequent dissociation at the nearest interface.

microscopic models ${ }^{37}$ or continuum approaches ${ }^{38-41}$ that use effective parameters in order to be computationally more efficient. For the purpose of this study of excitonic and bipolar transport, we use a simplified version of the model for BHJs presented in Ref. 42. Here the term "simplified" means that the influence of the bound electron-hole pair, as discussed, e.g., in Refs. 43-48, is neglected at the present level of abstraction. Typical exciton diffusion lengths are only of the order of $\sim 10 \mathrm{~nm}$ and, thus, much smaller than the device thickness. Therefore, we separate the transport of excitons in the $y$ direction parallel to the cell surface (i.e., to the next interface provided by the BHJ) from the $x$ direction of the electron and hole transport which is then normal to the cell's surface [cf. Fig. 2], similar as proposed by Minnaert et al. ${ }^{49}$

To include exciton diffusion to the next interface, we solve the one-dimensional diffusion equation for the exciton concentration $\chi$,

$$
0=D_{\chi} \frac{d^{2}}{d y^{2}} \chi-\frac{\chi}{\tau_{r}}+g_{\chi}
$$

as a function of the coordinate $y$ as indicated in Fig. 2. Here we assume that no dissociation can take place in the volume but only at the interfaces. The boundary conditions for the particle current densities per unit heterointerface area are assumed to be symmetric,

$$
j_{\chi}=\mp D_{\chi} \frac{d}{d y} \chi=S_{\chi} \chi-S_{\chi} \chi_{0} \frac{n p}{n_{i}^{2}},
$$

for $y= \pm w / 2$, where $w$ is the distance between two interfaces, $S_{\chi}$ is the surface recombination velocity for the excitons, and $n_{i}$ is the intrinsic carrier concentration. The solution for the exciton concentration is

$$
\chi=\left(A_{r}+A_{g}\right)\left[\cosh \left(y / L_{\chi}\right)\right]+g_{\chi} \tau_{r},
$$

where $L_{\chi}=\sqrt{\mu_{\chi} \tau_{r} k T / q}$. Equation (20) superimposes two contributions to the exciton population, namely, the optical generation, proportional to the exciton generation rate $g_{\chi}$,

$$
A_{g}=\frac{-S_{\chi} g_{\chi} \tau_{r}}{S_{\chi} \cosh \left(w / 2 L_{\chi}\right)+D_{\chi} / L_{\chi} \sinh \left(w / 2 L_{\chi}\right)},
$$

and the injection and extraction of excitons via the coupling to the free $e-h$ pairs,

$$
A_{r}=\frac{S_{\chi} \chi_{0} n p / n_{i}^{2}}{S_{\chi} \cosh \left(w / 2 L_{\chi}\right)+D_{\chi} / L_{\chi} \sinh \left(w / 2 L_{\chi}\right)},
$$

which is thus proportional to $n p / n_{i}^{2}$. In order to calculate the coupling between free $e-h$ pairs and excitons, we need to get rid of the $y$ dependence since we want to have a onedimensional model in the end.

Therefore, we replace the differential equation [Eq. (18)] together with its boundary condition [Eq. (19)] by a differential equation,

$$
0=-\frac{\bar{\chi}}{\tau_{r}}-\frac{\bar{\chi}}{\tau_{D}}+\frac{\chi_{0}}{\tau_{D}} \frac{n p}{n_{i}^{2}}+g_{\chi},
$$

that no longer contains any dependencies on a spatial coordinate or any transport terms. The information about exciton generation, diffusion, and dissociation, as determined by solving Eqs. (18) and (19), is now contained in the value of the average exciton concentration $\bar{\chi}$ and the value of the dissociation lifetime $\tau_{D}$. We average the concentration of excitons by integrating over the coordinate $y$ and receive $\bar{\chi}$ $=\bar{\chi}_{g}+\bar{\chi}_{r}$ with the two components,

$$
\bar{\chi}_{g}=g_{\chi} \tau_{r}\left[1-\frac{2 L_{\chi} S_{\chi} \sinh \left(w / 2 L_{\chi}\right) / w}{S_{\chi} \cosh \left(w / 2 L_{\chi}\right)+D_{\chi} / L_{\chi} \sinh \left(w / 2 L_{\chi}\right)}\right]
$$

and

$$
\bar{\chi}_{r}=\chi_{0} \frac{n p}{n_{i}^{2}} \frac{2 L_{\chi} S_{\chi} \sinh \left(w / 2 L_{\chi}\right) / w}{S_{\chi} \cosh \left(w / 2 L_{\chi}\right)+D_{\chi} / L_{\chi} \sinh \left(w / 2 L_{\chi}\right)} .
$$

Note that in the term $n p / n_{i}^{2}$ in Eq. (25), the voltage $V$ is hidden. For flat quasi-Fermi levels, the ratio $n p / n_{i}^{2}$ $=\exp (q V / k T)$, while for finite mobilities and depth dependent quasi-Fermi levels, the ratio $n p / n_{i}^{2}$ has to be calculated from solving the continuity equations for electrons and holes.

Again, the first component $\bar{\chi}_{g}$ accounts for the optically generated part of excitons and the second component $\bar{\chi}_{r}$ for the excitons that arise from injection of $e-h$ pairs. To be consistent with the steady-state condition of Eq. (23) and with the already determined average exciton concentration $\bar{\chi}=\bar{\chi}_{g}+\bar{\chi}_{r}$, the lifetime turns out to be

$$
\tau_{D}=\tau_{r}\left[\frac{w}{2 S_{\chi} \tau_{r}}+\frac{w}{2 L_{\chi}} \operatorname{coth}\left(w / 2 L_{\chi}\right)-1\right] .
$$

This result implies that the bulk heterojunction solar cell is included as a special case in our model as given by Eqs. (1)-(7). The only necessary modifications are setting $\mu_{\chi}(x)$ $=0$ in Eq. (1) and the calculation of the dissociation lifetime $\tau_{D}$ with the help of Eq. (26). Since separation of free charge carriers in BHJ solar cells is usually accomplished by using contact materials with different work functions, these devices fall under our category of $p-i-n$-junction solar cells. Since we do not consider the $p$-type and the $n$-type layers explicitly, the boundary conditions for free charge carriers and the electric field in the absorber are the same for the BHJ model and our standard $p-i$ - $n$-model. 


\section{Reciprocity between electroluminescence and quantum efficiency}

In the SQ limit of high mobilities and thus flat quasiFermi levels, the application of a voltage $V$ to the junction of the solar cell leads to an excess emission of photons given by

$$
\phi_{\mathrm{em}}(E)=A(E) \phi_{\mathrm{bb}}(E)\left[\exp \left(\frac{q V}{k T}\right)-1\right],
$$

where $A(E)$ denotes the cell absorptance dependent on photon energy $E$. The spectral dependence of the black body radiation emitted into the half sphere above the solar cell is given by

$$
\phi_{\mathrm{bb}}(E)=\frac{2 \pi E^{2}}{h^{3} c^{2}} \frac{1}{[\exp (E / k T)-1]} \approx \frac{2 \pi E^{2}}{h^{3} c^{2}} \exp \left(\frac{-E}{k T}\right),
$$

where $h$ is Planck's constant and $c$ is the vacuum speed of light. Equation (27) directly follows from Kirchhoff's law stating that absorptance and emissivity of a body are equal for each energy and spherical angle.

The SQ theory derives from Eq. (27) the saturation current density,

$$
J_{0, \mathrm{SQ}}=q \int_{0}^{\infty} A(E) \phi_{\mathrm{bb}}(E) d E .
$$

By the postulate that all absorbed photons generate one electron-hole pair and that all electron-hole pairs contribute to the short circuit current density, we obtain the maximum short circuit current density,

$$
J_{\mathrm{sc}, \mathrm{SQ}}=q \int_{0}^{\infty} A(E) \phi_{\mathrm{sun}}(E) d E,
$$

where $\phi_{\text {sun }}$ denotes the spectral photon flux arriving from the sun at the cell's surface.

For finite mobilities and nonconstant quasi-Fermi levels, the link between emission and absorption in a body must be extended to also cover injection and extraction of carriers or excitons. If transport in the absorber is described by a linear differential equation, the Donolato theorem, ${ }^{50-55}$ connecting injection of minorities in the dark and extraction of photogenerated carriers under illumination, is valid. Combining the two detailed balance pairs, absorption-collection (for the photovoltaic situation) and injection-emission (for the LED situation), a reciprocity relation between external photovoltaic quantum efficiency $Q_{e}$ and electroluminescent emission $\phi_{\mathrm{em}}$ has been derived, stating that ${ }^{24}$

$$
\phi_{\mathrm{em}}(E)=Q_{e}(E) \phi_{\mathrm{bb}}(E)\left[\exp \left(\frac{q V}{k T}\right)-1\right] .
$$

Equation (31) implies that the information contained in the EL spectrum equals that in the quantum efficiency. However, the multiplication with the black body spectrum, which decays exponentially for higher energies, weighs the quantum efficiency in such a way that the quantum efficiency can be derived experimentally from the EL emission only in the energetic region around the band gap. ${ }^{56-58}$
The equations for the radiative saturation current density,

$$
J_{0, \mathrm{rad}}=q \int_{0}^{\infty} Q_{e}(E) \phi_{\mathrm{bb}}(E) d E,
$$

and the short circuit current density,

$$
J_{\mathrm{sc}}=q \int_{0}^{\infty} Q_{e}(E) \phi_{\mathrm{sun}}(E) d E,
$$

follow in analogy to Eqs. (29) and (30) by exchanging quantum efficiency and absorptance. The radiative open circuit voltage,

$$
V_{\mathrm{oc}}=\frac{k T}{q} \ln \left(\frac{J_{\mathrm{sc}}}{J_{0, \mathrm{rad}}}+1\right),
$$

will then also depend on the quantum efficiency and thus on collection and injection of carriers and excitons. This is because the different spectral dependence of $\phi_{\mathrm{bb}}(E)$ and $\phi_{\text {sun }}(E)$ in Eqs. (32) and (33) will weigh the different spectral parts of $Q_{e}(E)$ differently. However, the resulting changes will be small because the ratio $J_{\mathrm{sc}} / J_{0, \text { rad }}$ enters only logarithmically into $V_{\mathrm{oc}}$.

The fill factor $\mathrm{FF}=\max (-J V) /\left(J_{\mathrm{sc}} V_{\mathrm{oc}}\right)$ of the currentvoltage curves under illumination, in the simplest case, is a function of the normalized open circuit voltage $u_{\mathrm{oc}}$ $=V_{\mathrm{oc}} /\left(n_{\mathrm{id}} k T / q\right)$, where $n_{\mathrm{id}}$ is the diode ideality factor. ${ }^{59}$ However, this is true only as long as the collection of carriers is a voltage-independent process. In Sec. III B we will discuss cases where voltage-dependent collection causes the FF to decrease although the open circuit voltage remains virtually unaffected. Voltage-dependent carrier collection violates also the assumptions required for the derivation of the reciprocity relation [Eq. (31)]. With our model, we can compare EL emission and the external quantum efficiency, which allows us to test whether or not a given configuration is compatible with the electro-optical reciprocity. We explicitly investigate the validity of reciprocity in Sec. III C and check whether a connection between this validity and the fill factor losses due to voltage-dependent collection (see Sec. III B) exists.

\section{RESULTS}

\section{A. Excitonic and bipolar photocurrent}

For all following simulations, we use a set of constant parameters, which are a thickness $d=300 \mathrm{~nm}$, the absorption coefficient of $\mathrm{ZnPc}$ as a typical organic absorber material (taken from Fig. 4 in Ref. 60), an optical generation profile, and a photon recycling scheme calculated according to Ref. 61 that both result from a Lambertian cell surface, an intrinsic carrier concentration $n_{i}=10^{3} \mathrm{~cm}^{-3}$, and an equilibrium built-in voltage of $V_{\mathrm{bi}, 0}=1.3 \mathrm{~V}$. Assuming a radiative lifetime of $\tau_{r}=200 \mu \mathrm{s}$, the equilibrium concentration of excitons follows from Eq. (4) as $\chi_{0}=4.4 \times 10^{-3} \mathrm{~cm}^{-3}$. All simulations are carried out in the radiative limit, i.e., there is no nonradiative recombination allowed. The other parameters, especially the mobilities and the dissociation lifetime, are varied in the following simulations. 


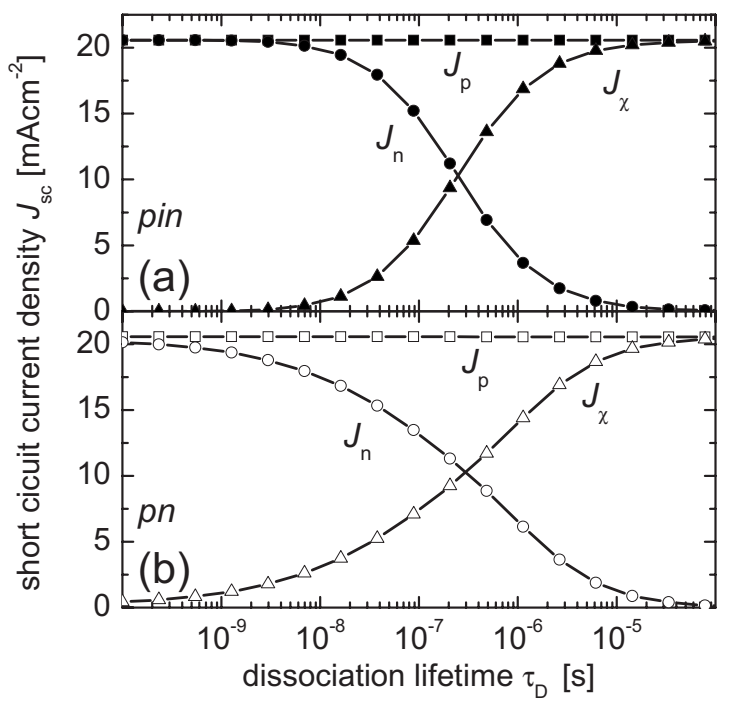

FIG. 3. Short circuit current density as a function of the bulk dissociation lifetime $\tau_{D}$ for the case of a (a) $p$ - $i$ - $n$-type and (b) $p n$-type devices. Efficient coupling of excitons to charge carriersi.e., low $\tau_{D}$-leads to bipolar transport, while an inefficient coupling forces the current to be transported by excitons to the contact. Since the electron contact is the only interface active for dissociation of excitons, the sum of electron current $J_{n}$ (flow of electrons injected from the absorber into the front contact) and the exciton current $J_{\chi}$ (flow of electrons injected from the absorber by interface dissociation of an exciton) always equals the hole current $J_{p}$ (flow of holes injected from the absorber into the back contact). The parameters used are (a) $\mu_{\chi}=\mu_{n}=\mu_{p}=10^{3} \mathrm{~cm}^{2} \mathrm{~V}^{-1} \mathrm{~s}^{-1}$ and $S_{n}$ $=S_{\chi n}=10^{6} \mathrm{~cm} \mathrm{~s}^{-1}$ and (b) $\mu_{\chi}=10^{-1} \mathrm{~cm}^{2} \mathrm{~V}^{-1} \mathrm{~s}^{-1}, \quad \mu_{n}=\mu_{p}$ $=10^{4} \mathrm{~cm}^{2} \mathrm{~V}^{-1} \mathrm{~s}^{-1}, S_{n}=10^{10} \mathrm{~cm} \mathrm{~s}^{-1}$, and $S_{\chi n}=50 \mathrm{~cm} \mathrm{~s}^{-1}$.

First, we show that a continuous transition from predominantly bipolar transport to predominantly excitonic transport is possible when varying the coupling between excitons and free charge carriers defined by the dissociation lifetime $\tau_{D}$. Since we are only interested in the dominant charge separation mechanism, we focus first on the short circuit situation. Because we assume only the left contact $(x=0)$ to be active in exciton dissociation, the total current equals the hole current $j_{p}(d)=j_{\chi}(0)+j_{n}(0)$ at the right contact $(x=d)$ as defined by Eq. (14). In order to be able to distinguish between electron current and excitonic current, we need to define the currents at the absorber side of the electron contact, i.e., at $x=0^{+}$. We distinguish between electrons injected into the contact from the reservoir of electrons in the absorber and electrons injected into contact, following a dissociation of an exciton. The former is denoted as electron current $j_{n}\left(0^{+}\right)$ $=S_{n}^{*} n_{j}-S_{n} n_{b}$ [see Eq. (11)] and the latter as exciton current $j_{\chi}\left(0^{+}\right)=H_{n} n_{j} p_{b}-S_{\chi n} \chi[$ see Eq. (8)].

Figure 3 shows how a variation in the dissociation lifetime in the range $10^{-4}>\tau_{D}>10^{-10} \mathrm{~s}$ for the case of a $p$ - $i$-n-type cell [Fig. 3(a)] and a pn-type cell [Fig. 3(b)] affects the excitonic contribution $J_{\chi}=-q j_{\chi}\left(0^{+}\right)$and the bipolar contributions $J_{n}=-q j_{n}\left(0^{+}\right)$and $J_{p}=q j_{p}(d)$ to the photocurrent. Note that by the fact that we have chosen the $n$-type contact (left in Fig. 1) to be the only contact active in dissociating excitons we have $J=J_{p}=J_{\chi}+J_{n}$. Figure 3(a) shows that for a short dissociation lifetime $\tau_{D}<10^{-8} \mathrm{~s}$, the solar cell behaves like a classical $p$ - $i$ - $n$-type device, where the exciton is split into free carriers already in the volume of the absorber. These free carriers then travel via drift-diffusion transport to their respective contacts, where they contribute to $j_{n}\left(0^{+}\right)$and $j_{p}(d)$. In order to achieve this bipolar transport, exciton dissociation and transport of free carriers must be faster than exciton diffusion and interface dissociation and faster than exciton recombination. Since, we chose our equilibrium concentrations such that $n_{i} \gg \chi_{0}$ holds, for high mobilities $\mu_{\chi}=\mu_{n}=\mu_{p}=10^{3} \mathrm{~cm}^{2} \mathrm{~V}^{-1} \mathrm{~s}^{-1}$ and high interface dissociation and collection velocities $S_{n}, S_{\chi n}>10^{5} \mathrm{~cm} \mathrm{~s}^{-1}$ of free carriers and excitons, free carriers will always be able to carry larger currents. In contrast, for dissociation lifetimes $\tau_{D}>10^{-5} \mathrm{~s}$ interfacial exciton dissociation is responsible for the total current at the electron contact, i.e., energy transport via excitons is now more efficient than the slow bulk dissociation with subsequent fast bipolar transport. This limit corresponds to the situation of an "excitonic" solar cell as defined by Refs. 21 and 22 .

The situation for the pn-type solar cell in Fig. 3(b) is qualitatively very similar. There is also a transition from the bipolar to the excitonic operation mode visible with an intersection between both contributions at dissociation lifetimes around $\tau_{D} \approx 2 \times 10^{-7} \mathrm{~s}$ just like for the pin case. However, there are two distinct differences. The first is the larger extension of the transition region and the second is that the mobilities had to be changed compared to the $p$ - $i$ - $n$-type case. For equal and high mobilities of both excitons and free carriers, as was the case for Fig. 3(a), the whole current would have been transported by excitons no matter how fast they dissociate. This becomes obvious when considering that although $n_{i} \gg \chi_{0}$ holds, the concentration of minority carriers $n_{i}^{2} / N_{A}=1.2 \times 10^{-5} \mathrm{~cm}^{-3} \ll \chi_{0}$, where $N_{A}$ is the acceptor concentration. Note that we chose the doping for the $p n$-type cell such that the voltage, where minorities (electrons) become equal to the doping concentration, corresponds to the built-in voltage $V_{\mathrm{bi}, 0}=1.3 \mathrm{~V}$ for the $p$ - $i$ - $n$-case, which was chosen to be just above the radiative open circuit voltage. Since there are more excitons than minority carriers available to transport the energy, we needed to make the mobilities and/or the interface dissociation velocities unequal to get a transition from excitonic to bipolar transport. Thus, we chose $\mu_{\chi}$ $=10^{-1} \mathrm{~cm}^{2} \mathrm{~V}^{-1} \mathrm{~s}^{-1}, \quad \mu_{n}=\mu_{p}=10^{4} \mathrm{~cm}^{2} \mathrm{~V}^{-1} \mathrm{~s}^{-1}, \quad S_{n}$ $=10^{10} \mathrm{~cm} \mathrm{~s}^{-1}$, and $S_{\chi n}=50 \mathrm{~cm} \mathrm{~s}^{-1}$ in Fig. 3(b). Note that in case of the $p$ - $i$ - $n$-type solar cell [Fig. 3(a)], the choice of the dissociation and collection velocities does not affect the photocurrent and its distribution into $J_{\chi}$ and $J_{n}$ as long as $S_{n}, S_{\chi n}>10^{5} \mathrm{~cm} \mathrm{~s}^{-1}$. A series of simulations of other interesting situations for the current collection in excitonic and bipolar solar cells can be found elsewhere. ${ }^{62}$

Besides the coupling constant $\tau_{D}$ between excitons and free carriers, also the equilibrium concentrations of excitons and free carriers affect the dominant charge separation pathway [cf. Eq. (5)]. Note that the ratio between those equilibrium concentrations is determined by the binding energy $\Delta E_{B}=E_{C}-E_{\text {exc }}$ of the exciton. In order to keep the energy $E_{\text {exc }}$ of the excitons and, therefore, the overall absorptance as well as the maximum short circuit current constant, we shift the energy $E_{C}$ by a relative amount $\delta E_{C}$ as illustrated in Fig. 4(a). A lowering of $E_{C}$ at constant $E_{\text {exc }}$ means that the bind- 


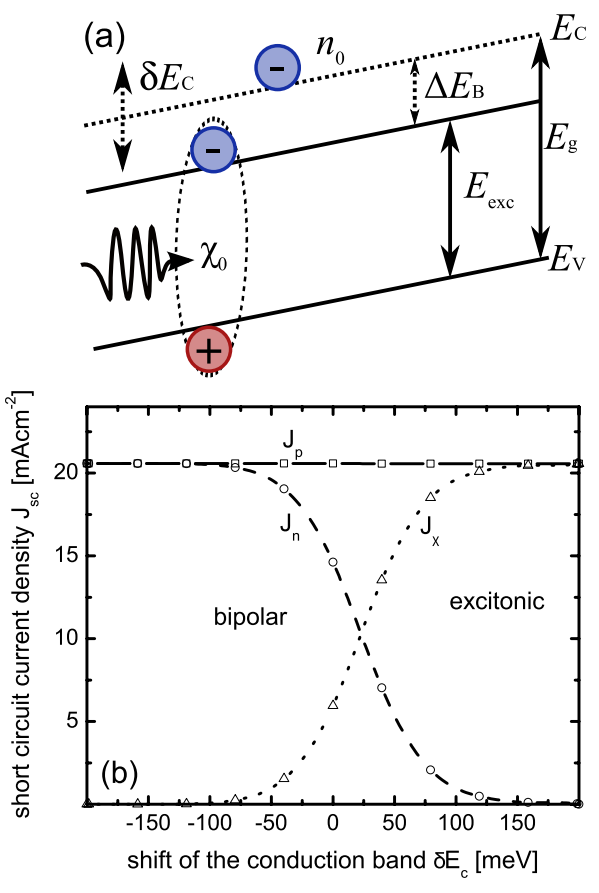

FIG. 4. (Color online) (a) Band diagram showing that a relative shift $\delta E_{C}$ of the conduction band is equivalent to a change in exciton binding energy. (b) Variations in the short circuit current density resulting from the relative shift $\delta E_{C}$ of the conduction band for the free electrons in the volume for the case of a $p$ - $i$-n-type device. Shifting the conduction band leads to a change in electron equilibrium concentration $n_{0}$ and thus-for constant $R_{\chi}=4.4$ $\times 10^{-2} \mathrm{~cm}^{3} \mathrm{~s}^{-1}$ and $\chi_{0}$ - to a change in the dominating transport mechanism. Note that the choice of $R_{\chi}$ leads to $\tau_{D}=10^{-7} \mathrm{~s}$ for $\delta E_{C}=0$, such that direct comparison is possible with Fig. 3(a). Shifting the conduction band up lowers $n_{0}$ and makes transport via excitons become more favorable. In order not to affect the extraction of excitons and carriers at the contacts and the built-in voltage, the equilibrium concentrations in the contacts are not changed and mark the zero position in the $\delta E_{C}$ axis.

ing energy $\Delta E_{B}$ of the exciton becomes smaller and vice versa.

Figure 4(b) shows a simulation where the conductionband edge and consequently the equilibrium concentration of electrons in the volume of the device varies. Note that the conduction band at the contact is not changed in order to avoid intermixing of additional effects due to enhanced or deteriorated extraction of excitons and carriers at the contact.
If the conduction band is lifted up, the equilibrium concentration of electrons is diminished, and thus, the photocurrent becomes predominantly excitonic. In contrast, when the conduction-band energy is lowered, there are more electrons in the absorber available to carry the current, and thus, the balance shifts in the direction of the bipolar current.

\section{B. Current-voltage curves}

Starting with the limit of high mobilities, we discuss in the following how restrictions for excitonic and/or carrier transport affect the shape of the $J / V$ curve and the solar cell parameters short circuit current density $J_{\mathrm{sc}}$ and FF. For our calculations, we focus on the case of $p-i-n$-type solar cells since this type of device shows specific nonlinear features associated with the fact that recombination of electrons with holes contains the product of hole and electron concentrations. We discuss $p-i-n$ solar cells in both the excitonic as well as in the bipolar limit as defined by Fig. 3(a).

The following computations use four different combinations of parameters, as summarized in Table I. Configurations $\mathrm{A}$ and $\mathrm{B}$ are normal pin devices where both excitonic and bipolar transport is possible. Configuration $\mathrm{A}$ is a device with slow coupling between excitons and free carriers $\left(\tau_{D}\right.$ $=10^{-4} \mathrm{~s}$ ) where we want to focus on the effect of small exciton mobilities. Configuration B is a mainly bipolar device with an efficient coupling between excitons and free carriers where we want to investigate the effect of low bipolar mobilities. Configurations $\mathrm{C}$ and D are BHJ solar cells with zero exciton mobility in $x$ direction as discussed in Sec. II C. Configuration $\mathrm{C}$ is the $\mathrm{BHJ}$ equivalent of configuration $\mathrm{A}$ in the sense that high mobilities for electrons and holes are assumed in order to concentrate on the influence of variations in the exciton mobility $\mu_{\chi}$ on the overall device performance. In the frame of the present effective BHJ model, we only take into account perpendicular exciton transport to the next internal heterointerface, which we summarize with a modified effective exciton dissociation lifetime $\tau_{D}$. For instance, $\tau_{D}=3 \times 10^{-4}$ s corresponds to $\mu_{\chi}=10^{-8} \mathrm{~cm}^{2}(\mathrm{~V} \mathrm{~s})^{-1}$, $S_{\chi}=10^{10} \mathrm{~cm} / \mathrm{s}$, and $w=10 \mathrm{~nm}$ because of Eq. (26). Complementarily, configuration D covers the case of efficient exciton dissociation and creation of free carriers in a BHJ. Here, we are interested in the investigation of effects resulting from decreasing electron and hole mobilities.

Figure 5 shows simulated current/voltage curves for configuration A, i.e., a solar cell with a fixed electron and hole

TABLE I. Mobilities $\mu_{n, p}$ of free carriers and $\mu_{\chi}$ of excitons as well as the bulk dissociation lifetime $\tau_{D}$ for the three discussed configurations of nonideal solar cells. In case of configurations $\mathrm{C}$ and $\mathrm{D}$, the dissociation lifetime $\tau_{D}$ is calculated via the equation for bulk heterojunction solar cells [Eq. (26)]. The parameters used to obtain these values are for configuration C: a lifetime $\tau_{r}=200 \mu$ s, an interface dissociation velocity $S_{\chi}=10^{10} \mathrm{~cm} / \mathrm{s}$, and an interface distance $w=10 \mathrm{~nm}$. Furthermore, we assumed that the exciton diffusion length follows from $L_{\chi}=\sqrt{\mu \tau_{r} k T / q}$. For configuration D, we used $L_{\chi}=10$ and $w=100 \mathrm{~nm}$. All other parameters were the same as for configuration C.

\begin{tabular}{lcccc}
\hline \hline & Configuration A & Configuration B & Configuration C & Configuration D \\
\hline Carrier mobility $\mu_{n, p}\left[\mathrm{~cm}^{2}(\mathrm{~V} \mathrm{~s})^{-1}\right]$ & $10^{3}$ & $0.1-10^{3}$ & $10^{3}$ & $0.1-10^{3}$ \\
Exciton mobility $\mu_{\chi}\left[\mathrm{cm}^{2}(\mathrm{~V} \mathrm{~s})^{-1}\right]$ & $10^{-8}-10^{3}$ & $10^{-5}$ & 0 (in $x$ direction) & 0 (in $x$ direction) \\
& & & $10^{-8}-10^{-1}$ (in $y$ direction) & $2 \times 10^{-5}$ \\
Dissociation lifetime $\tau_{D}(s)$ & $10^{-4}$ & $10^{-10}$ & $3 \times 10^{-11}-3 \times 10^{-4}$ & $2 \times 10^{-7}$ \\
\hline \hline
\end{tabular}




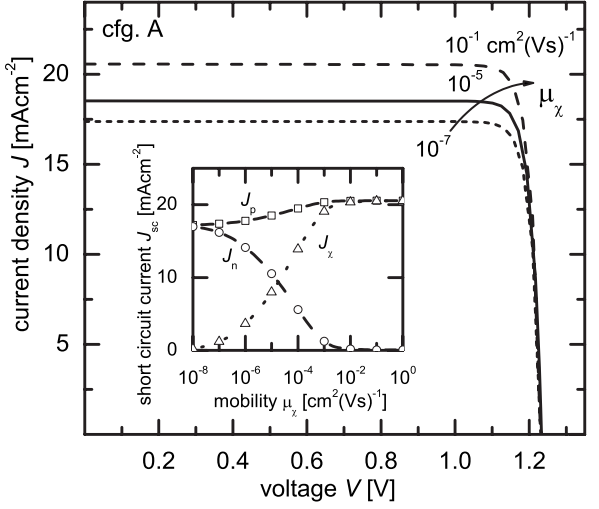

FIG. 5. Simulation of current-voltage curves for a solar cell with a fixed electron and hole mobility $\mu_{n, p}=10^{3} \mathrm{~cm}^{2}(\mathrm{~V} \mathrm{~s})^{-1}$ and a fixed dissociation lifetime $\tau_{D}=10^{-4} \mathrm{~s}$ (configuration A in Table I). The parameter is the exciton mobility $\mu_{\chi}=10^{-1}, 10^{-5}, 10^{-7} \mathrm{~cm}^{2}(\mathrm{~V} \mathrm{~s})^{-1}$ which leads to a decrease in short circuit current while keeping the fill factor the same. In the inset, the short circuit current density $J_{\mathrm{sc}}$ (solid line) and the contributions to the current density $J_{n}$ and $J_{\chi}$ from electrons (dashed line) and excitons (dotted line) are depicted as functions of exciton mobility. Starting from the high mobility limit $\mu_{\chi}>10^{-1} \mathrm{~cm}^{2}(\mathrm{~V} \mathrm{~s})^{-1}$ a decreasing exciton mobility leads to a decrease in $J_{\chi}$, which is partly compensated by an increase in $J_{n}$, leading to a saturation of $J_{\text {sc }}$ for low exciton mobilities $\mu_{\chi}$.

mobility $\mu_{n / p}=10^{3} \mathrm{~cm}^{2}(\mathrm{~V} \mathrm{~s})^{-1}$ and a fixed dissociation lifetime $\tau_{D}=10^{-4} \mathrm{~s}$. This long dissociation lifetime corresponds to a situation that is excitonic for high exciton mobilities $\mu_{\chi}$ as shown in Fig. 3(a). The solid line corresponds to an exciton mobility $\mu_{\chi}=10^{-5} \mathrm{~cm}^{2}(\mathrm{~V} \mathrm{~s})^{-1}$, while the dashed and the dotted line correspond to an exciton mobility $\mu_{\chi}$ $=10^{-1} \mathrm{~cm}^{2}(\mathrm{~V} \mathrm{~s})^{-1}$ (dashed) and $\mu_{\chi}=10^{-7} \mathrm{~cm}^{2}(\mathrm{~V} \mathrm{~s})^{-1}$ (dotted). The dashed curve corresponding to the high exciton mobility $\mu_{\chi}=10^{-1} \mathrm{~cm}^{2}(\mathrm{~V} \mathrm{~s})^{-1}$ equals the SQ limit as defined by Eqs. (29) and (30). Such that, every absorbed photon contributes one elementary charge to the short circuit current and the radiative losses are given by the equation for $J_{0, \mathrm{SQ}}$ [Eq. (29)]. For lower mobilities, the decreased collection of excitons leads to the situation where the quantum efficiency becomes smaller than the absorptance and both $J_{0, \text { rad }}$ and $J_{\text {sc }}$ decrease. The open circuit voltage [see Eq. (34)] as well as the fill factor remains almost unaffected by the change in exciton mobility.

A more detailed analysis of the short circuit current density $J_{\text {sc }}$ (solid line) and the contributions to the current density $J_{n}$ and $J_{\chi}$ from electrons (dashed line) and excitons (dotted line) is depicted in the inset as a function of exciton mobility. Starting from the high mobility limit $\mu_{\chi}$ $>10^{-1} \mathrm{~cm}^{2}(\mathrm{~V} \mathrm{~s})^{-1}$, a decreasing exciton mobility leads to a decrease in $J_{\chi}$, which is partly compensated by an increase in $J_{n}$, leading to a saturation of $J_{\mathrm{sc}}$ for low exciton mobilities $\mu_{\chi}$. Since the dissociation lifetime $\tau_{D}=10^{-4} \mathrm{~s}=100 \mu \mathrm{s}$ is similar to the radiative lifetime $\tau_{r}=200 \mu \mathrm{s}$, not all excitons dissociate faster than they recombine, which explains why the decreasing exciton current is not completely compensated by the electron current.

Figure 6 shows a simulation of configuration B, i.e., a $p$ - $i$ - $n$-type device with a fixed exciton mobility $\mu_{\chi}$

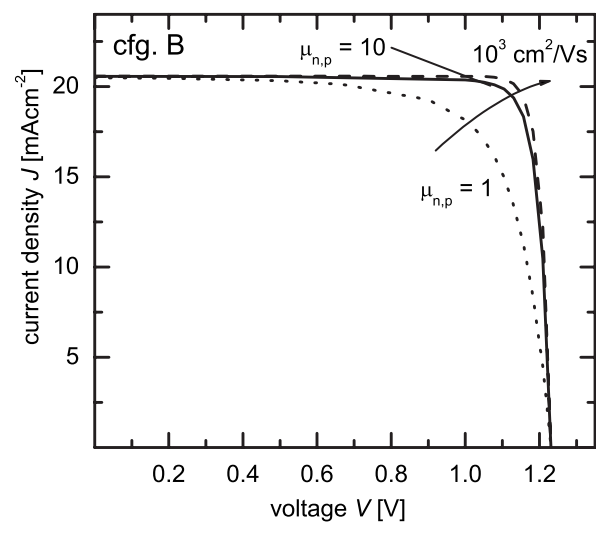

FIG. 6. Simulation of current-voltage curves for a solar cell with a fixed exciton mobility $\mu_{\chi}=10^{-5} \mathrm{~cm}^{2}(\mathrm{~V} \mathrm{~s})^{-1}$ and a fixed dissociation lifetime $\tau_{D}=10^{-10} \mathrm{~s}$ (configuration B from Table I). The parameter is the carrier mobility $\mu_{n, p}=10^{0}, 10^{1}, 10^{3} \mathrm{~cm}^{2}(\mathrm{~V} \mathrm{~s})^{-1}$ which leads to a decrease in fill factor while keeping the short circuit current roughly the same. The solid line corresponds to the parameter configuration B from Table I. In the inset, the fill factor is depicted as a function of the carrier mobility $\mu_{n, p}$.

$=10^{-5} \mathrm{~cm}^{2}(\mathrm{~V} \mathrm{~s})^{-1}$ and a fixed dissociation lifetime $\tau_{D}$ $=10^{-10} \mathrm{~s}$. At high mobilities of free carriers $\left[\mu_{n, p}\right.$ $\left.\geq 10^{3} \mathrm{~cm}^{2}(\mathrm{~V} \mathrm{~s})^{-1}\right]$, this short dissociation lifetime corresponds to a bipolar photocurrent under short circuit conditions as shown in Fig. 3(a). Here, we vary the carrier mobility $\mu_{n, p}=10^{0}, 10^{1}, 10^{3} \mathrm{~cm}^{2}(\mathrm{~V} \mathrm{~s})^{-1}$. The decreasing carrier mobility $\mu_{n, p}$ leads to a decay in fill factor and in short circuit current density $J_{\mathrm{sc}}$. However, contrary to configuration A (Fig. 5), the fill factor loss is by far more dominant than that in $J_{\mathrm{sc}}$. This is because for low charge carrier mobilities in $p$-i-n-type solar cells, the effect of the built-in field becomes decisive. Since the charge carriers, unlike the excitons, react on the decrease in the built-in field resulting by application of forward bias voltage $V$, the photocurrent decreases with increasing $V$. The voltage dependence of carrier collection reduces the fill factor. Therefore, the fill factor is strongly influenced by the carrier mobilities $\mu_{n, p}$ although the open circuit voltage is not dependent on $\mu_{n, p}$ in Fig. 6 .

Instead of discussing the current-voltage curves of configurations $\mathrm{C}$ and $\mathrm{D}$ in the same detail as A and $\mathrm{B}$, we will now focus on the main results. Thus, Fig. 7 summarizes the effects of either low exciton or low electron and hole mobilities on the short circuit current density $J_{\mathrm{sc}}$ and fill factor FF of configurations A-D. Figures 7(a) and 7(b) show that the decrease in both $J_{\mathrm{sc}}$ and FF resulting from decreasing the charge carrier mobilities $\mu_{n, p}$ is virtually identical for BHJ and non-BHJ devices. This lack of difference between two basically different types of devices results from the fact that the bottleneck in both situations is the mobility of the charge carriers which act in both devices in the same way. The fill factor loss shows up at mobilities $\mu_{n, p}<10^{2} \mathrm{~cm}^{2}(\mathrm{~V} \mathrm{~s})^{-1}$, while a loss in $J_{\mathrm{sc}}$ starts only for much lower mobilities $\mu_{n, p}<10^{0} \mathrm{~cm}^{2}(\mathrm{~V} \mathrm{~s})^{-1}$. This is because the voltage dependence of the carrier collection probability from the $300-\mathrm{nm}$ thick absorber shows up at high forward bias voltage $V$ first (leading to FF losses) before at even lower $\mu_{n, p}$ also carrier collection at $V=0$ is diminished (leading to losses in $J_{\mathrm{sc}}$ ). 


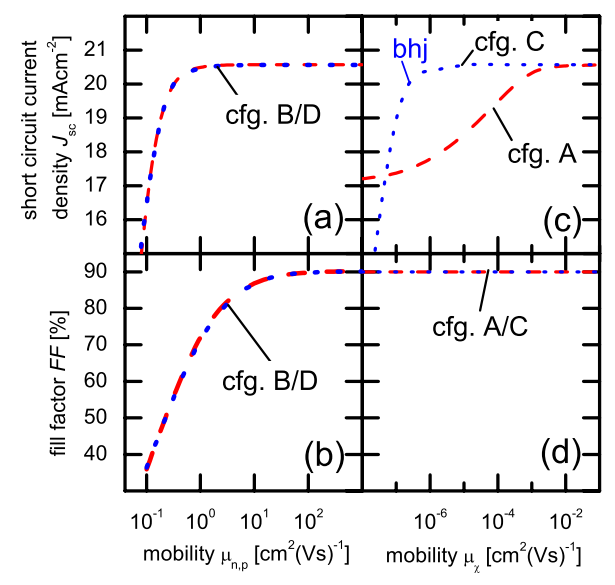

FIG. 7. (Color online) Summary of the effects of reduced bipolar and excitonic mobilities on short circuit current density $J_{\text {sc }}$ and fill factor FF. In (a) and (b) we used the parameters for configurations B (dashed line) and D (dotted line) except for the electron and hole mobility $\mu_{n, p}$ which is varied. We find a considerable decrease in fill factor for mobilities $\mu_{n, p}<10^{2} \mathrm{~cm}^{2}(\mathrm{~V} \mathrm{~s})^{-1}$, while a loss in $J_{\mathrm{sc}}$ starts only for even lower mobilities $\mu_{n, p}<10^{0} \mathrm{~cm}^{2}(\mathrm{~V} \mathrm{~s})^{-1}$. Both, the BHJ and the non-BHJ solar cells show the same dependence of $J_{\text {sc }}$ and FF on mobility. In (c) and (d), we used the parameters for configurations A (dashed line) and $\mathrm{C}$ (dotted line), except for the varied exciton mobility. For the non-BHJ cell (configuration A), $J_{\mathrm{sc}}$ is reduced for exciton mobilities $\mu_{\chi}<10^{-3} \mathrm{~cm}^{2}(\mathrm{~V} \mathrm{~s})^{-1}$. For the $\mathrm{BHJ}$ cell (dotted line) the decrease in $J_{\mathrm{sc}}$ starts only at $\mu_{\chi}$ $<10^{-6} \mathrm{~cm}^{2}(\mathrm{~V} \mathrm{~s})^{-1}$, where the exciton mobility is too low to reach the heterointerface. The fill factor is in both cases unaltered because the electron and hole mobilities are high enough to warrant voltageindependent collection.

Figures 7(c) and 7(d) show the effect of decreasing exciton mobility $\mu_{\chi}$ while keeping electron and hole mobilities at a high value of $\mu_{n, p}=10^{3} \mathrm{~cm}^{2}(\mathrm{~V} \mathrm{~s})^{-1}$. Due to the different definitions of exciton mobility in BHJ and non-BHJ solar cells, there is a considerable difference in the dependence of $J_{\text {sc }}$ on exciton mobility. For the non-BHJ solar cell, we obtain the result, which was already depicted in the inset of Fig. 5, namely, a decrease in photocurrent exciton mobilities $\mu_{\chi}$ $<10^{-3} \mathrm{~cm}^{2}(\mathrm{~V} \mathrm{~s})^{-1}$. This decline saturates at a level of about $83 \%$ of the maximum $J_{\mathrm{sc}}$. This plateau results from the fact that the loss in excitonic current is partly compensated by an increase in electron and hole currents. For BHJ solar cells, this bypass is not possible because exciton dissociation takes place exclusively at the heterointerface. Nevertheless, the decrease in $J_{\mathrm{sc}}$ for the BHJ case starts only at $\mu_{\chi}$ $<10^{-6} \mathrm{~cm}^{2}(\mathrm{~V} \mathrm{~s})^{-1}$, i.e., 3 orders of magnitude below the critical value for the non-BHJ case. This larger allowance for low exciton mobilities is the basic advantage of the BHJ resulting from the much smaller path length of excitons to the separating heterointerface compared to the non-BHJ case. The fill factor as shown in Fig. 7(d) is independent from the exciton mobility in both cases. This finding is expected because changes in the electrical field cannot alter the collection of excitons. As a result the decline of photocurrent is voltage independent.

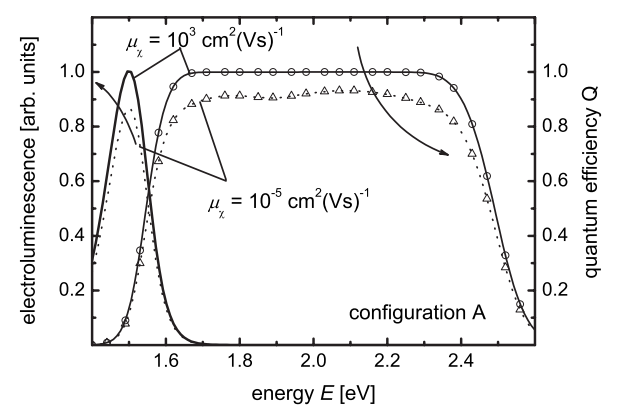

FIG. 8. Quantum efficiency (symbols), EL spectrum, and quantum efficiency calculated from the EL according to Eq. (31) for an ideal cell with high carrier mobilities (solid lines) and for a cell with the parameters according to configuration A defined in Table I (dotted line). For both devices the reciprocity theorem is valid.

\section{Electroluminescence and quantum efficiency}

For the four different parameter configurations of Table I, we investigate the validity of the reciprocity theorem defined by Eq. (31). Figure 8 illustrates the physical content of Eq. (31). The electroluminescence (EL) emission simulated with the model described above allows us to derive the quantum efficiency via Eq. (31). For the case of high mobilities the quantum efficiency derived from EL (solid line) agrees with the directly simulated quantum efficiency (open circles). Thus, for perfect transport both excitonic and bipolar solar cells follow the reciprocity theorem ${ }^{24}$ no matter whether the device is build as $p$-i-n-type or $p n$-type device.

Proceeding now to less ideal devices, we investigate under which circumstances the reciprocity relation begins to lose its validity. Figure 8 also shows the EL spectrum (dotted line), the quantum efficiency calculated from the EL spectrum via the reciprocity theorem (dotted), and the quantum efficiency (open triangles) of the solar cell with parameter configuration A $\left[\mu_{n, p}=10^{3} \mathrm{~cm}^{2}(\mathrm{~V} \mathrm{~s})^{-1}, \quad \mu_{\chi}\right.$ $=10^{-5} \mathrm{~cm}^{2}(\mathrm{~V} \mathrm{~s})^{-1}$, and $\left.\tau_{D}=10^{-4} \mathrm{~s}\right]$. Since this configuration leads to a relatively constant photocurrent and ideal recombination current, as shown by the mobility independent fill factor in Figs. 5 and 7(d), also the reciprocity is valid.

Figure 9 summarizes the validity of the reciprocity theorem by comparing the directly simulated quantum efficiency $Q_{e, \text { dir }}$ (diamonds) and the quantum efficiency $Q_{e, \text { EL }}$ calculated from the simulation of the EL (solid lines) for the case of configurations A-D. For configurations $\mathrm{A}$ and $\mathrm{C}$ as shown in Figs. 9(a) and 9(c), the electron and hole mobilities are high and thus the reciprocity is valid although the quantum efficiency is below unity in the saturation regime. Voltageindependent collection and injection of carriers at high bipolar mobilities thus implies both high and constant fill factors [see Fig. 7(d)] and the validity of the reciprocity relation $\left(Q_{e, \mathrm{dir}}=Q_{e, \mathrm{EL}}\right)$.

Figures 9(b) and 9(d) show that for configurations B and $\mathrm{D}$, the reciprocity is no longer valid $\left(Q_{e, \mathrm{dir}} \neq Q_{e, \mathrm{EL}}\right)$. We learned from Figs. 6 and 7(b) that a mobility of $\mu_{n, p}$ $=10 \mathrm{~cm}^{2}(\mathrm{~V} \mathrm{~s})^{-1}$ already leads to a small fill factor loss of around $\Delta \mathrm{FF}=3 \%$ compared to the high mobility case. In consequence, we also find a small deviation of quantum efficiency $Q_{e, \text { dir }}$ (diamonds) and quantum efficiency $Q_{e, \mathrm{EL}}$ from 


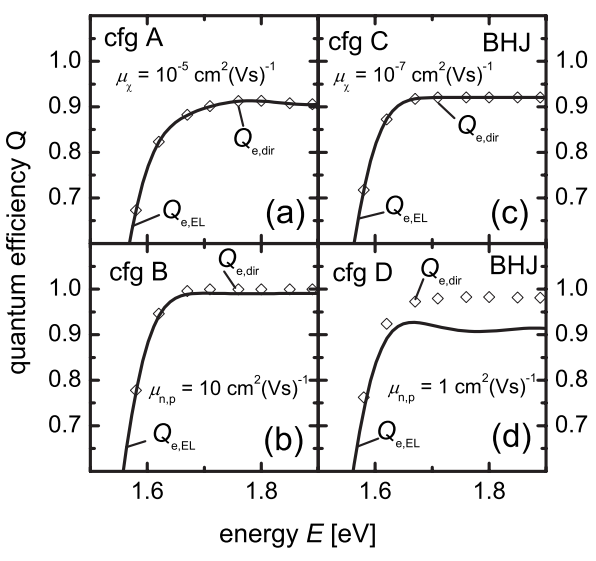

FIG. 9. Simulation of the quantum efficiency $Q_{e, \text { dir }}$ (diamonds) compared with the simulation of the electroluminescence spectrum from which the quantum efficiency $Q_{e, \mathrm{EL}}$ (solid line) is calculated using Eq. (31) for the case of configurations (a) A, (b) B, (c) C, and (d) D. For cases (a) and (c), the electron and hole mobilities are high $\left[\mu_{n, p}=10^{3} \mathrm{~cm}^{2}(\mathrm{~V} \mathrm{~s})^{-1}\right]$ and thus collection and injection of carriers is voltage independent and the reciprocity is valid. For case (b), the low bipolar mobility leads to a small deviation between $Q_{e, \text { dir }}$ and $Q_{e, \text { EL }}$. For case (d), the even lower mobility leads to a clearly visible deviation between $Q_{e, \text { dir }}$ and $Q_{e, \mathrm{EL}}$.

EL (solid line), as shown in Fig. 9(b). Figure 9(d) shows the case of a BHJ solar cell with a mobility of $\mu_{n, p}$ $=1 \mathrm{~cm}^{2}(\mathrm{~V} \mathrm{~s})^{-1}$ (configuration D). From Fig. 7(b), we learn that the fill factor decays by roughly $\Delta \mathrm{FF}=19 \%$ compared to the high mobility case. Thus, the deviation between $Q_{e \text {,dir }}$ (diamonds) and $Q_{e, \mathrm{EL}}$ (solid line) in Fig. $9(\mathrm{~d})$ is much stronger than in Fig. 9(b).

Note here that the voltage dependence of collection and thus injection makes the EL spectrum a function of applied voltage. The voltage used for all EL spectra in Figs. 8 and 9 is $V=1 \mathrm{~V}$. Using higher (lower) voltages would increase (decrease) the difference between direct quantum efficiency simulated at $V=0 \mathrm{~V}$ and quantum efficiency calculated from EL.

\section{Bulk heterojunction cells: Role of band offsets and morphology}

Up to this point, we have considered radiative recombination as the only loss mechanism. This implies that departures from the SQ limit are due to low mobility effects showing up in the fill factor and the short circuit current density, whereas the open circuit voltage is determined by radiative recombination only. However, $V_{\text {oc }}$ of all real solar cells is controlled by nonradiative recombination. In BHJ solar cells nonradiative recombination at the heterointerfaces is an important loss process determining the open circuit voltage. As a direct consequence, the band offset $\Delta E_{C}$ between conduction band of donor and acceptor molecules and the morphology, ${ }^{63}$ i.e., the average distance $w$ between two heterointerfaces, become decisive parameters for the efficiency of the BHJ solar cell. Interestingly, both parameters bear a trade-off between optimizing dissociation of excitons favored by low values of $w$ and large band offsets $\Delta E_{C}$ and the minimization of re- combination losses, requiring large distances $w$ and band offsets as small as possible. The following will provide a quantitative treatment of these effects.

It is useful to describe the exciton transport to the interface in terms of a collection efficiency, ${ }^{42}$

$$
f_{c}=\frac{\bar{\chi}_{g}}{\left(g_{\chi} \tau_{D}\right)}=\frac{2 L_{\chi} S_{\chi}}{w} \frac{\sinh \left(w / 2 L_{\chi}\right)}{S_{\chi} \sinh \left(w / 2 L_{\chi}\right)+D_{x} / L_{\chi} \cosh \left(w / 2 L_{\chi}\right)} .
$$

The collection efficiency is equivalent to the effective generation rate $\bar{\chi}_{g} / \tau_{D}$ [cf. Eqs. (24) and (26)] of free carriers in our BHJ model in short circuit and under illumination, normalized to the generation rate $g_{\chi}$ for excitons. For very high exciton diffusion lengths $L_{\chi} \gg w$, the collection efficiency saturates at its maximum. This maximum is usually $f_{c}=1$ if exciton dissociation at the interface is efficient. For ratios $w / 2 L_{\chi}<1$, the collection efficiency decreases below its saturation level.

Let us first focus on the band offset $\Delta E_{C}$ at the interface and assume that the transport of excitons to the interface and the collection of electrons and holes are efficient. Efficient transport of excitons to the interface means for our model that the exciton diffusion length is much higher than the distance to the next interface, i.e., $L_{\chi} \gg w$. Efficient collection of electrons and holes means that the mobilities of the free charge carriers are so high that the quasi-Fermi level splitting is constant throughout the device. If these two requirements are reasonable assumptions, both transport of carriers and transport of excitons do not limit the photocurrent and the only limiting process is the dissociation of excitons at the interface. Then, the short circuit current density $J_{\text {sc }}$ normalized to its maximum $J_{\mathrm{sc}, \mathrm{SQ}}$ will be equal to the collection efficiency $f_{c}$ in the limit of high exciton diffusion lengths $L_{\chi} \gg w$. From Eq. (35) follows

$$
\frac{J_{\mathrm{sc}}}{J_{\mathrm{sc}, \mathrm{SQ}}}=\lim _{w / L_{\chi} \rightarrow 0} f_{c}=\frac{1}{1+w /\left(2 S_{\chi} \tau_{r}\right)} .
$$

At the interface, detailed balance leads to an equation similar to Eqs. (6) and (7), defining the exciton dissociation at the contacts. In thermal equilibrium, the recombination of electrons in the acceptor phase and holes in the donor phase equals the dissociation of excitons, leading to

$$
H_{\chi} n_{0 A} p_{0 D}=S_{\chi} \chi_{0},
$$

where $H_{\chi}$ defines the recombination rate and $S_{\chi}$ defines the dissociation rate. The equilibrium concentrations $n_{0 A}$ and $p_{0 D}$ of electrons in the acceptor and holes in the donor will depend on the band gap of the respective material. Figure 10 shows a scheme of the heterojunction between donor and acceptor together with the equilibrium concentrations depending on the conduction-band and valence-band energy $E_{\mathrm{CA}}$ and $E_{\mathrm{VA}}$ in the acceptor and $E_{\mathrm{CD}}$ and $E_{\mathrm{VD}}$ in the donor. Let us now vary the conduction-band energy $E_{\mathrm{CA}}$ in the acceptor, we will have a change in the left-hand side of $\mathrm{Eq}$. (37) since $n_{0 A} \sim \exp \left(\Delta E_{C} / k T\right)$, where $\Delta E_{C}=E_{\mathrm{CD}}-E_{\mathrm{CA}}$ is the difference in conduction-band energy between donor and acceptor. If everything else is kept constant, then a high band offset $\Delta E_{C}$ implies a low band gap of the acceptor and, thus, 


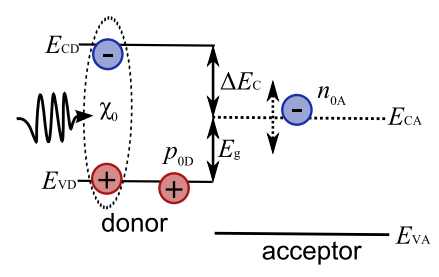

FIG. 10. (Color online) Schematic drawing of the heterointerface between donor and acceptor in a $\mathrm{BHJ}$ solar cell. By varying the conduction-band energy $E_{\mathrm{CA}}$ of the acceptor, the equilibrium concentration $n_{0 A}$ of electrons in the acceptor, the band gap $E_{g}$ as well as the band offset $\Delta E_{C}$ in the conduction band between donor and acceptor are changed.

a high equilibrium concentration $n_{0 A}$. The validity of Eq. (37) requires that the dissociation rate $S_{\chi}$ also changes with the band offset since $\chi_{0}$ stays constant. The latter is a useful assumption since changing $\chi_{0}$ would also mean that the band gap of the donor and therewith the absorption in the solar cell would change. Thus

$$
S_{\chi}=S_{00} \exp \left(\Delta E_{C} / k T\right)
$$

holds, where $S_{00}$ is a proportionality factor in units of a velocity. Equation (38) means that the dissociation is more efficient if the band offset is high. For the normalized short circuit current density, this exponential dependence of the dissociation velocity on the band offset implies that

$$
\frac{J_{\mathrm{sc}}}{J_{\mathrm{sc}, \mathrm{SQ}}}=\frac{1}{1+\frac{w}{2 S_{00} \tau_{r}} \exp \left(-\Delta E_{C} / k T\right)}
$$

holds.

Now, we need to consider the dependence of the recombination current on the band offset, i.e., we have to take into account nonradiative interface-recombination. For a device with an extremely high amount of internal surfaces as is necessary in organic BHJ solar cells, it is reasonable to assume the recombination at these interfaces dominates the recombination current. ${ }^{64}$ Under this assumption, the saturation current density $j_{0}$ (notably, a particle current density by unit area of the heterojunction interface) will be proportional to the equilibrium carrier concentrations. Since the hole concentration in the donor remains unchanged, we write

$$
j_{0}=j_{00} \exp \left(\Delta E_{C} / k T\right),
$$

with $j_{00}$ being a proportionality factor with the unit of a particle current density. Since a higher conduction-band offset $\Delta E_{C}$ implies a smaller band gap $E_{g}$ (as defined in Fig. 10), the recombination increases. To obtain the electrical short circuit current density $J_{0}$ per unit solar cell area for the geometry sketched in Fig. 2, we use

$$
J_{0}=q j_{0} \frac{d}{w}=q j_{00} \exp \left(\Delta E_{C} / k T\right) \frac{d}{w}=J_{00} \exp \left(\Delta E_{C} / k T\right) .
$$

Note that we have assumed all charge carrier mobilities to be infinite and therefore the saturation current density $J_{0}$ is pro-

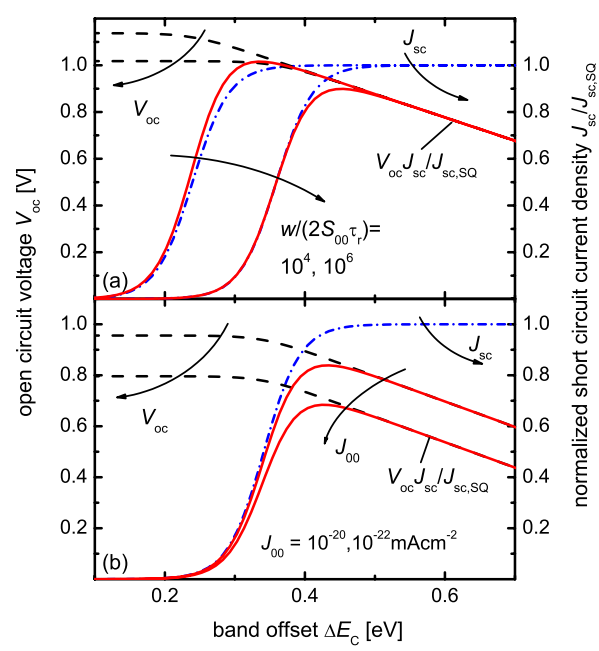

FIG. 11. (Color online) Simulation of short circuit current density $J_{\mathrm{sc}}$ (normalized to the maximum $J_{\mathrm{sc}, \mathrm{SQ}}$ ), open circuit voltage $V_{\mathrm{oc}}$, and the product $V_{\mathrm{oc}} J_{\mathrm{sc}} / J_{\mathrm{sc}, \mathrm{SQ}}$ as functions of the band offset $\Delta E_{c}$ in a BHJ-solar cell using the analytical Eqs. (39)-(41). The excitonic transport to the next heterointerface is assumed to be efficient $L_{\chi} / w \gg 1$ and thus the only relevant parameters are (a) the ratio $w /\left(2 S_{00} \tau_{r}\right)=10^{4}, 10^{6}$ and (b) $J_{00}=10^{-22}, 10^{-20} \mathrm{~mA} \mathrm{~cm}{ }^{-2}$. A high band offset generally favors $J_{\mathrm{sc}}$ since the exciton dissociation is made much more favorable than its inverse process (recombination of two free carriers forming an exciton). A low band offset favors $V_{\mathrm{oc}}$ since the energy loss at the heterointerface is minimized and band gaps (and minority carrier concentration) are kept as high (and low) as possible to ensure a low recombination current.

portional to the device thickness $d$ and inversely proportional to the separation distance $w$ between the heterointerfaces.

Figure 11 shows the open circuit voltage $V_{\mathrm{oc}}$, the normalized short circuit current density $J_{\mathrm{sc}} / J_{\mathrm{sc}, \mathrm{SQ}}$, and the relative dependence of the product $V_{\mathrm{oc}} J_{\mathrm{sc}} / J_{\mathrm{sc}, \mathrm{SQ}}$ as functions of the conduction-band offset $\Delta E_{C}$. The product $V_{\mathrm{oc}} J_{\mathrm{sc}} / J_{\mathrm{sc}, \mathrm{SQ}}$ serves as a measure for the efficiency $\eta$ since $\eta=F F J_{\mathrm{sc}} V_{\mathrm{oc}}$; but it allows us to make the result independent of the value of the photocurrent $J_{\mathrm{sc}, \mathrm{SQ}}$ in the limit of high mobilities and perfect collection, i.e., $f_{c}=1$.

We have only two possible parameters, which change the dependence of $V_{\mathrm{oc}}, J_{\mathrm{sc}} / J_{\mathrm{sc}, \mathrm{SQ}}$ and $V_{\mathrm{oc}} J_{\mathrm{sc}} / J_{\mathrm{sc}, \mathrm{SQ}}$ on the band offset: The first parameter, which is varied in Fig. 11(a), is the product $w /\left(2 S_{00} \tau_{r}\right)$, and the second, being varied in Fig. 11(b), is the prefactor $J_{00}$ of the saturation current density. Both Figs. 11(a) and 11(b) show that we obtain a maximum of the product $V_{\mathrm{oc}} J_{\mathrm{sc}} / J_{\mathrm{sc}, \mathrm{SQ}}$ with respect to the band offset $\Delta E_{C}$. This maximum is in the range $0.3<\Delta E_{C}<0.5 \mathrm{eV}$ mostly depending on $w /\left(2 S_{00} \tau_{r}\right)$.

The second parameter we study is the distance $w$ between two heterointerfaces. For the short circuit current density, we now have to abandon the assumption of efficient transport to the interface since the collection of excitons heavily depends on the distance to the next interface. For the normalized $J_{\mathrm{sc}}$, then holds

$$
\frac{J_{\mathrm{sc}}}{J_{\mathrm{sc}, \mathrm{SQ}}}=f_{c}=\frac{2 L_{\chi} S_{\chi}}{w} \frac{\sinh \left(w / 2 L_{\chi}\right)}{S_{\chi} \sinh \left(w / 2 L_{\chi}\right)+\frac{D_{\chi}}{L_{\chi}} \cosh \left(w / 2 L_{\chi}\right)} .
$$




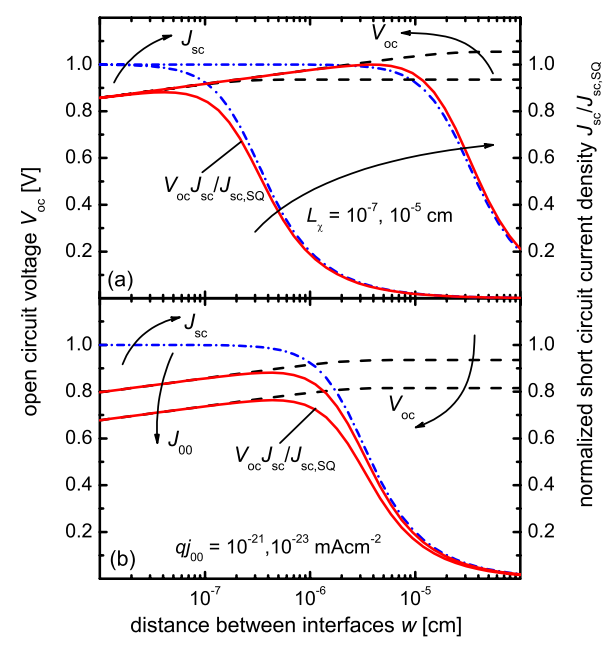

FIG. 12. (Color online) Simulation of short circuit current density $J_{\mathrm{sc}}$ (normalized to the maximum $J_{\mathrm{sc}, \mathrm{SQ}}$ ), open circuit voltage $V_{\mathrm{oc}}$, and the product $V_{\mathrm{oc}} J_{\mathrm{sc}} / J_{\mathrm{sc}, \mathrm{SQ}}$ as functions of the distance between two heterointerfaces in a BHJ solar cell using the analytical Eqs. (41) and (42). We vary (a) the exciton diffusion length $L_{\chi}$ $=10^{-7}, 10^{-5} \mathrm{~cm}$ and (b) $q j_{00}=10^{-23}, 10^{-21} \mathrm{~mA} \mathrm{~cm}{ }^{-2}$. A high distance between interfaces generally favors $V_{\text {oc }}$ since interface recombination is minimized, while a low distance favors $J_{\text {sc }}$ due to more efficient diffusion of excitons to the next interface.

Figure 12 shows the open circuit voltage $V_{\mathrm{oc}}$, the normalized short circuit current density $J_{\mathrm{sc}} / J_{\mathrm{sc}, \mathrm{SQ}}$, and the relative dependence of the product $V_{\mathrm{oc}} J_{\mathrm{sc}} / J_{\mathrm{sc}, \mathrm{SQ}}$ as functions of the distance between two interfaces $w$. The values of device thickness and band offset used to calculate $J_{0}$ are $d$ $=100 \mathrm{~nm}$ and $\Delta E_{C}=0.4 \mathrm{eV}$.

Now, we have more free parameters than before since we use a more complicated ansatz for the normalized short circuit current density. As parameters for Fig. 12, we chose the exciton diffusion length $L_{\chi}$ and the prefactor $J_{00}$ of the saturation current density. The other parameters that were kept constant are the recombination lifetime $\tau_{r}=100 \mu$ s and the prefactor $S_{00}=1 \mathrm{~cm} / \mathrm{s}$ of the surface recombination velocity.

Figure 12(a) shows the variation in the exciton diffusion length $L_{\chi}$ leading to a varying optimum distance $w$ for a maximum product $V_{\mathrm{oc}} J_{\mathrm{sc}} / J_{\mathrm{sc}, \mathrm{SQ}}$. As shown in Fig. 12(b), for a constant exciton diffusion length $L_{\chi}=10 \mathrm{~nm}$ and varying $J_{00}$, the optimum distance stays roughly the same. The optimum is rather broad and peaks at distances of around $5 \mathrm{~nm}$, i.e., slightly smaller than the exciton diffusion length.

\section{CONCLUSIONS}

The present paper has introduced a detailed balance model for solar cells that includes transport and interaction of excitons, electrons, and holes. Due to the possibility to continuously shift the dominant transport mechanism from free carriers to excitons, the distinction between excitonic and classical solar cells, although useful for the analysis of practical devices, turns into limiting cases of the present general theory. Since the term excitonic solar cells encompasses mostly organic polymer/fullerene solar cells, we discuss a model to include specific features of bulk heterojunction solar cells in our general model. In order to take exciton diffusion to the internal donor/acceptor interface into account, we solve the diffusion equation for excitons analytically. These analytical solutions then serve as effective parameters for the numerical solution of the coupled transport equations in one dimension.

In the limit of high mobilities of excitons, electrons, and holes, the model reproduces the SQ efficiency limit, while for lower mobilities differences between excitonic and bipolar as well as $p$-i-n-type and $p n$-type solar cells become apparent. In bipolar $p-i-n$-type solar cells, low mobilities will lead to incomplete collection of carriers. Since carrier collection depends on the internal electric field, which changes with applied voltage, for low mobilities $p$ - $i$ - $n$-type solar cells show a voltage-dependent collection and injection of carriers. For cells with mainly excitonic transport, no voltage dependencies are apparent since exciton diffusion does not depend on the built-in field. The difference in voltage dependence is reflected in the validity of the fundamental reciprocity of photovoltaic quantum efficiency and electroluminescent emission of solar cells. For voltage-dependent collection and injection, the reciprocity is no longer valid, which is proven by the simulation of electroluminescence and quantum efficiency spectra.

While in the radiative limit, the open circuit voltage is always the same for a given absorption coefficient and thickness and is independent of transport; some important solar cell parameters only affect the simulation if nonradiative recombination limits the efficiency. As an example for the influence of nonradiative recombination on our detailed balance model, we discuss the effects of band offsets and the average distance between the heterointerfaces in bulk heterojunction solar cells. In both cases, there are competing effects on open circuit voltage $V_{\text {oc }}$ and short circuit current density $J_{\text {sc }}$ such that we always find an optimum for the product $V_{\mathrm{oc}} J_{\mathrm{sc}} / J_{\mathrm{sc}, \mathrm{SQ}}$ and consequently for the power conversion efficiency of the devices.

\section{ACKNOWLEDGMENTS}

The authors would like to thank C. Deibel (University Würzburg) and K. Taretto (Universidad Nacional del Comahue, Neuquén) for fruitful discussions. 
*Corresponding author: t.kirchartz@fz-juelich.de

${ }^{1}$ D. M. Chapin, C. S. Fuller, and G. L. Pearson, J. Appl. Phys. 25, 676 (1954).

${ }^{2}$ M. A. Green, Solar Cells: Operating Principles, Technology, and System Applications (University of New South Wales, Sydney, 1986), pp. 103-169.

${ }^{3}$ D. Ginley, M. A. Green, and R. Collins, MRS Bull. 33, 355 (2008).

${ }^{4}$ N. S. Lewis, Science 315, 798 (2007).

${ }^{5}$ R. F. Service, Science 319, 718 (2008).

${ }^{6}$ B. O'Regan and M. Grätzel, Nature (London) 353, 737 (1991).

${ }^{7}$ N. S. Sariciftci, L. Smilowitz, A. J. Heeger, and F. Wudl, Science 258, 1474 (1992).

${ }^{8}$ I. Gur, N. A. Fromer, M. L. Geier, and A. P. Alivisatos, Science 310, 462 (2005).

${ }^{9}$ W. U. Huynh, J. J. Dittmer, and A. P. Alivisatos, Science 295, 2425 (2002).

${ }^{10}$ W. J. E. Beek, M. M. Wienk, M. Kemerink, X. N. Yang, and R. A. J. Janssen, J. Phys. Chem. B 109, 9505 (2005).

${ }^{11}$ R. D. Schaller and V. I. Klimov, Phys. Rev. Lett. 92, 186601 (2004).

${ }^{12}$ P. Guyot-Sionnest, Nature Mater. 4, 653 (2005).

${ }^{13}$ A. Luque, A. Marti, and A. J. Nozik, MRS Bull. 32, 236 (2007).

${ }^{14}$ A. J. Nozik, Physica E 14, 115 (2002).

${ }^{15}$ V. I. Klimov, J. Phys. Chem. B 110, 16827 (2006).

${ }^{16}$ U. Bach, D. Lupo, P. Comte, J. E. Moser, F. Weissörtel, J. Salbeck, H. Spreitzer, and M. Grätzel, Nature (London) 395, 583 (1998).

${ }^{17}$ M. Grätzel, Nature (London) 414, 338 (2001).

${ }^{18}$ P. Wang, S. M. Zakeeruddin, J. E. Moser, M. K. Nazeeruddin, T. Sekiguchi, and M. Grätzel, Nature Mater. 2, 402 (2003).

${ }^{19}$ M. Law, L. E. Greene, J. C. Johnson, R. Saykally, and P. Yang, Nature Mater. 4, 455 (2005).

${ }^{20}$ L. Schmidt-Mende, U. Bach, R. Humphry-Baker, T. Horiuchi, H. Miura, S. Ito, S. Uchida, and M. Grätzel, Adv. Mater. 17, 813 (2005).

${ }^{21}$ B. A. Gregg and M. C. Hanna, J. Appl. Phys. 93, 3605 (2003).

${ }^{22}$ B. A. Gregg, J. Phys. Chem. B 107, 4688 (2003).

${ }^{23}$ W. Shockley and H. J. Queisser, J. Appl. Phys. 32, 510 (1961).

${ }^{24}$ U. Rau, Phys. Rev. B 76, 085303 (2007).

${ }^{25}$ A. Martí, J. L. Balenzategui, and R. F. Reyna, J. Appl. Phys. 82, 4067 (1997).

${ }^{26}$ W. van Roosbroeck and W. Shockley, Phys. Rev. 94, 1558 (1954).

${ }^{27}$ U. Rau, G. Kron, and J. H. Werner, J. Phys. Chem. B 107, 13547 (2003).

${ }^{28}$ G. Yu, J. Gao, J. C. Hummelen, F. Wudl, and A. J. Heeger, Science 270, 1789 (1995).

${ }^{29}$ C. J. Brabec, N. S. Sariciftci, and J. C. Hummelen, Adv. Funct. Mater. 11, 15 (2001).

${ }^{30}$ H. Hoppe and N. S. Sariciftci, J. Mater. Res. 19, 1924 (2004).

${ }^{31}$ F. Yang, M. Shtein, and S. R. Forrest, Nature Mater. 4, 37 (2005).

${ }^{32}$ P. W. M. Blom, V. D. Mihailetchi, L. J. A. Koster, and D. E. Markov, Adv. Mater. (Weinheim Ger.) 19, 1551 (2007).

${ }^{33}$ Y. Kim, S. Cook, S. M. Tuladhar, S. A. Choulis, J. Nelson, J. R.
Durrant, D. D. C. Bradley, M. Giles, I. McCulloch, C.-S. Ha, and M. Ree, Nature Mater. 5, 197 (2006).

${ }^{34}$ J. Peet, J. Y. Kim, N. E. Coates, W. L. Ma, D. Moses, A. J. Heeger, and G. C. Bazan, Nature Mater. 6, 497 (2007).

${ }^{35}$ J. Y. Kim, K. Lee, N. E. Coates, D. Moses, T.-Q. Nguyen, M. Dante, and A. J. Heeger, Science 317, 222 (2007).

${ }^{36}$ K. M. Coakley, Y. Liu, C. Goh, and M. D. McGehee, MRS Bull. 30, 37 (2005).

${ }^{37}$ R. A. Marsh, C. Groves, and N. C. Greenham, J. Appl. Phys. 101, 083509 (2007).

${ }^{38}$ S. Lacic and O. Inganäs, J. Appl. Phys. 97, 124901 (2005).

${ }^{39}$ L. J. A. Koster, E. C. P. Smits, V. D. Mihailetchi, and P. W. M. Blom, Phys. Rev. B 72, 085205 (2005).

${ }^{40}$ C. M. Martin, V. M. Burlakov, and H. E. Assender, Sol. Energy Mater. Sol. Cells 90, 900 (2006).

${ }^{41}$ C. M. Martin, V. M. Burlakov, H. E. Assender, and D. A. R. Barkhouse, J. Appl. Phys. 102, 104506 (2007).

${ }^{42}$ T. Kirchartz, B. E. Pieters, K. Taretto, and U. Rau, J. Appl. Phys. 104, 094513 (2008)

${ }^{43}$ V. D. Mihailetchi, L. J. A. Koster, J. C. Hummelen, and P. W. M. Blom, Phys. Rev. Lett. 93, 216601 (2004).

${ }^{44}$ D. W. Sievers, V. Shrotriya, and Y. Yang, J. Appl. Phys. 100, 114509 (2006).

${ }^{45}$ H. H. P. Gommans, M. Kemerink, J. M. Kramer, and R. A. J. Janssen, Appl. Phys. Lett. 87, 122104 (2005).

${ }^{46}$ L. J. A. Koster, V. D. Mihailetchi, R. Ramaker, and P. W. M. Blom, Appl. Phys. Lett. 86, 123509 (2005).

${ }^{47}$ L. J. A. Koster, V. D. Mihailetchi, and P. W. M. Blom, Appl. Phys. Lett. 88, 093511 (2006).

${ }^{48}$ J. D. Kotlarski, P. W. M. Blom, L. J. A. Koster, M. Lenes, and L. H. Slooff, J. Appl. Phys. 103, 084502 (2008).

${ }^{49}$ B. Minnaert, C. Grasso, and M. Burgelman, C. R. Chim. 9, 735 (2006).

${ }^{50}$ C. Donolato, Appl. Phys. Lett. 46, 270 (1985).

${ }^{51}$ K. Misiakos and F. A. Lindholm, J. Appl. Phys. 58, 4743 (1985).

${ }^{52}$ C. Donolato, J. Appl. Phys. 66, 4524 (1989).

${ }^{53}$ T. Markvart, IEEE Trans. Electron Devices 43, 1034 (1996).

${ }^{54}$ M. A. Green, J. Appl. Phys. 81, 269 (1997).

${ }^{55}$ U. Rau and R. Brendel, J. Appl. Phys. 84, 6412 (1998).

${ }^{56}$ T. Kirchartz, U. Rau, M. Kurth, J. Mattheis, and J. H. Werner, Thin Solid Films 515, 6238 (2007).

${ }^{57}$ T. Kirchartz and U. Rau, J. Appl. Phys. 102, 104510 (2007).

${ }^{58}$ T. Kirchartz, U. Rau, M. Hermle, A. W. Bett, A. Helbig, and J. H. Werner, Appl. Phys. Lett. 92, 123502 (2008).

${ }^{59}$ M. A. Green, Solar Cells: Operating Principles, Technology, and System Applications (University of New South Wales, Sydney, 1986), p. 80.

${ }^{60}$ J. Rostalski and D. Meissner, Sol. Energy Mater. Sol. Cells 63, 37 (2000).

${ }^{61}$ J. Mattheis, J. H. Werner, and U. Rau, Phys. Rev. B 77, 085203 (2008).

${ }^{62}$ T. Kirchartz and U. Rau, Thin Solid Films 516, 7144 (2008).

${ }^{63}$ H. Hoppe and N. S. Sariciftci, J. Mater. Chem. 16, 45 (2006).

${ }^{64}$ L. J. A. Koster, V. D. Mihailetchi, and P. W. M. Blom, Appl. Phys. Lett. 88, 052104 (2006). 